\title{
Grammatische Traditionen im Vergleich: die Satzdefinition in der deutschen und italienischen Grammatikschreibung
}

\author{
Von Patrizio Malloggi, Università di Pisa
}

\section{Einleitung}

Untersuchungsgegenstand des vorliegenden Artikels ist die Satzdefinition aus kontrastiver Sicht (Deutsch-Italienisch). Ausgangspunkt ist die Feststellung, dass der Satzbegriff eines der umstrittensten Konzepte der Sprachwissenschaft ist, über dessen Bestimmung große Uneinigkeit herrscht; dies zeigen allein schon die ca. dreihundert Versuche zu deutschsprachigen Satzdefinitionen'. In der neueren Linguistik reichen die Versuche von der formalen strukturalistischen Bestimmung als größte selbstständige syntaktische Form über funktionale Bestimmungen als Redeeinheiten mit kommunikativer Funktion bis hin zu rein theorieimmanenten Bestimmungen, bei denen der Satz durch die Beschreibung der Regularitäten syntaktischer Konstruktionen definiert wird $^{2}$. Trotz dieser bemerkenswerten Anzahl von Definitionsversuchen konnte bisher kein Ansatz alle Kriterien erfüllen, was sich nicht zuletzt daran zeigt, dass sich noch keiner dieser Ansätze in der Forschung durchgesetzt hat ${ }^{3}$. Dies lässt erkennen, dass es schwer ist, eine erschöpfende Satzdefinition zu finden. Dass es eine wissenschaftlich allgemein akzeptierte Satzdefinition bisher nicht gibt, kann ein Hindernis für die Grammatikvermittlung in der Fremdsprachendidaktik bedeuten. Es fehlt ein allgemein gültiges theoretisches Bezugsmodell in der Grammatikschreibung, auf dessen Grundlage Reflexionen über Aufbau (z.B. Stellung des finiten Verbs) und Struktur (Reihenfolge der Konstituenten) des deutschen Satzes entwickelt werden können.

Die Satzdefinition wird in diesem Beitrag auf der Grundlage der neueren Grammatiken des Deutschen bzw. Italienischen untersucht. Es ist Tradition der Grammatikschreibung, den Satz als eine grammatische Basiseinheit zu sehen. Beschäftigt man sich jedoch genauer mit Grammatiken, so fällt auf,

1 Vgl. etwa John Ries: Was ist ein Satz? Prag 1931. Eugen Seidel: Geschichte und Kritik der wichtigsten Satzdefinitionen. Jena 1935.

2 Vgl. Gisela Zifonun/Ludger Hoffmann/Bruno Strecker: Grammatik der deutschen Sprache. Berlin 1997, S. 86.

3 Vgl. Beat Louis Müller: Der Satz. Definition und sprachtheoretischer Status. Tübingen 1985, S. 23 (=Reihe Germanistische Linguistik 57) 
dass der Satz entweder gar nicht definiert wird ${ }^{4}$, dass die Definition zirkulär ist (d.h. jeder Satz hat ein Subjekt und ein Prädikat) und/oder dass es so viele Satzdefinitionen wie grammatiktheoretische Ansätze gibt ${ }^{5}$.

Anliegen dieses Aufsatzes ist es festzustellen, ob die Grammatikschreibung des Gegenwartsdeutschen und -italienischen dem Satz jeweils dieselbe Definition zuschreibt und welche Gemeinsamkeiten bzw. Unterschiede die aufgeführten Satzdefinitionen aufweisen können. Dabei werden die Definitionen des Satzes aus den ausgewählten Grammatiken systematisch klassifiziert und miteinander verglichen. Bei der kontrastiv ausgerichteten Untersuchung der Satzdefinition wird ferner festgestellt, ob der Satzbegriff einzelsprachlich- bzw. sprachübergreifend-zentriert zu konzipieren ist.

Der vorliegende Artikel setzt sich aus drei Abschnitten zusammen: Im ersten Abschnitt werden die Datengrundlage beschrieben und die Untersuchungsmethode erklärt. Der zweite Abschnitt ist der kontrastiv ausgerichteten Untersuchung der Satzdefinitionen in der Grammatikschreibung des Gegenwartsdeutschen bzw. -italienischen gewidmet. Im dritten Abschnitt werden die Ergebnisse der Untersuchung zusammenfassend aufbereitet. Hier wird auch der abschließende Vergleich hinsichtlich der Satzdefinition in beiden Zielsprachen angestellt sowie das Fazit gezogen. Der abschließende Vergleich setzt sich zum Ziel, eine wissenschaftlich allgemeinverbindliche Satzdefinition für beide Vergleichssprachen zu formulieren. Der Definitionsvorschlag von Satz soll als tertium comparationis für kontrastiv ausgerichtete Studien zur Syntax des Satzes und zur Grammatikvermittlung in der Fremdsprachendidaktik dienen.

\section{Datengrundlage und Untersuchungsmethode}

Als Datengrundlage dient eine Sammlung von Satzdefinitionen, die in der Grammatikschreibung des Gegenwartsdeutschen bzw. -italienischen vorkommen. Die grundlegende Bedeutung des grammatischen Ansatzes bei der Satzdefinition wird insbesondere von Heringer unterstrichen:

„Eine Grammatik soll eine Beschreibung der Struktur der Sätze einer Sprache sein. Ein bestimmter Satz einer Sprache muss nach der Grammatik möglich sein, d.h. er muss eine Struktur haben, die die Grammatik zulässt. Deshalb ist die Struktur eines Satzes eine geordnete Teilmenge der Grammatik“".

4 Peter Eisenberg: Grundriss der deutschen Grammatik. Stuttgart 1989.

5 Peter Schlobinski: Funktionale Grammatik und Sprachbeschreibung. Eine Untersuchung zum gesprochenen Deutsch sowie zum Chinesischen. Wiesbaden 1992, S. 114.

6 Hans Jürgen Heringer: Theorie der deutschen Syntax. München 1970, S. 230. (=Linguistische Reihe 1) 
Für die Zielsetzung dieses Artikels wurden ausgewählte Grammatiken herangezogen, d.h. solche, die die geschriebene Standardsprache der Gegenwart beschreiben und dabei auf dem aktuellen Forschungsstand fußen: für das Deutsche gelten die Grammatiken von Heringer ${ }^{7}$, Zifonun et al. ${ }^{8}$, Engel ${ }^{9}$ und Duden $^{10}$; für das Italienische orientiert sich die Beschreibung an den Grammatiken von Renzi ${ }^{11}$, Salvi/Vanelli12 ${ }^{12}$ Schwarze ${ }^{13}$ und Serianni ${ }^{14}$.

Anzumerken ist, dass die hier vorgestellten Satzdefinitionen eigens für die geschriebene Sprache entwickelt worden sind und entsprechend nicht ohne weiteres auf die gesprochene Sprache übertragen werden können. Die Satzdefinitionen unterscheiden sich nämlich in der Grammatikographie der gesprochenen Sprache und der Schriftsprache voneinander. Auf diese Unterscheidung werde ich in diesem Aufsatz nicht näher eingehen, da ich mich ausschließlich auf die geschriebene Sprache konzentriere.

Die gesammelten Satzdefinitionen werden zunächst aus einer einzelsprachlichen Perspektive untersucht und die entsprechenden typischen Merkmale festgestellt; dann werden die berücksichtigten Satzdefinitionen sprachübergreifend analysiert, mit dem Ziel, die gemeinsamen Merkmale in den Vordergrund zu rücken.

\section{Satzdefinition im deutsch-italienischen Sprachvergleich}

Seit dem 19. Jahrhundert wird der Satz aus unterschiedlichen Perspektiven beschrieben. In den folgenden Abschnitten werden die in den ausgewählten gängigen Grammatiken beider Vergleichssprachen vorkommenden Definitionen des Satzes systematisch untersucht.

\subsection{Der Satzbegriff in der Grammatikschreibung des Gegenwartsdeutschen}

Die Darstellung der Satzdefinitionen in der gängigen Grammatikschreibung des Deutschen erfolgt in chronologischer Reihenfolge und nimmt den Zeitraum

7 Hans Jürgen Heringer: Lesen, lehren, lernen: Eine rezeptive Grammatik des Deutschen. Tübingen 1989.

8 Zifonun et al., Grammatik der deutschen Sprache (wie Anm. 2).

9 Ulrich Engel: Deutsche Grammatik. München 2009.

10 Duden: Die Grammatik: unentbehrlich für richtiges Deutsch. Band 4. Berlin ${ }^{9} 2016$.

11 Lorenzo Renzi/Giampaolo Salvi/Anna Cardinaletti (a cura di): Grande grammatica italiana di consultazione. Volume I. La frase. I sintagmi nominale e preposizionale. Bologna 2001.

12 Giampaolo Salvi/ Laura Vanelli: Nuova grammatica italiana. Bologna 2004.

13 Christoph Schwarze: Grammatik der italienischen Sprache. Tübingen 1995.

14 Luca Serianni: Grammatica Italiana. Italiano comune e lingua letteraria. Novara 2006. 
von den 90er Jahren bis zur Gegenwart in Anspruch. In der „rezeptiven Grammatik“15 von Heringer ist der Satz stark formal ausgeprägt: „Der Satz bestehe aus einer Verbalphrase mit finitem Verb als Kern" ${ }^{" 16}$. Bei Heringer wird der Satz von innen heraus, d.h. vom Verb aus betrachtet; dabei wird festgestellt, in welcher Beziehung die nicht-verbalen Elemente zum Verb stehen. Daraus ergibt sich eine Definition des Satzes, den man als verbzentriert beschreiben kann. Der Satz wird nämlich als Ergebnis der vom Verb abhängigen syntaktischen Konstruktionen konzipiert.

Zifonun et al. definieren den Satz aus einer formalen und funktionalen Perspektive. Ihre Definition beschreibt Sätze als ,übergreifende Konstruktionsformen, die mindestens aus einem finiten Verb und dessen - unter strukturellen und kontextuellen Gesichtspunkten - notwendigen Komplementen bestehen ${ }^{\text {“117 }}$. Die Autoren der IDS-Grammatik ${ }^{18}$ rücken folgende Merkmale des Satzes in den Vordergrund:

(a) Der Satz muss aus (mindestens) einem finiten Verb bzw. einem Prädikat ${ }^{19}$ bestehen (formale bzw. syntaktische Perspektive).

(b) Der Satz weist eine semantische bzw. kommunikative Funktion auf, die dadurch zustande kommt, dass das finite Verb durch seine Argumente gesättigt wird. Es handelt sich hierbei um den Ausdruck eines Sachverhaltsentwurfs (funktionale Perspektive)

Die unter (a) und (b) angegebenen Merkmale des Satzes ergeben sich aus dem Zusammenspiel zwischen dem finiten Verb und seinen Komplementen bzw. Argumentausdrücken.

Engel greift in seiner Deutschen Grammatik aus gleicher Perspektive auf den Satz zu. Ihm zufolge weisen Sätze eine spezifische Grundstruktur auf, die darin besteht, dass ,,jeder Satz ein finites Verb enthält. Als satzbegründendes Element ist das finite Verb der wichtigste Teil des Satzes. [...]. Es bildet den Kern eines engeren Konstrukts, in dem die Satzglieder als seine Satelliten

15 Unter der Bezeichnung, ,rezeptive Grammatik“ wird eine Grammatik verstanden, die in erster Linie auf die Anforderungen und Bedürfnisse von Rezipienten zugeschnitten sein soll. Dabei ist die rezeptive Perspektive entscheidend, die die formalen Strukturen auf ihre Leistungskraft für den Verstehensprozess hin beschreibt (vgl. Thomas Schröder: Die Handlungsstruktur von Texten. Ein integrativer Beitrag zur Texttheorie. Tübingen 2003, S. 96.

16 Heringer: Lesen, lehren, lernen, S. 56 (wie Anm. 7).

17 Zifonun et al., Grammatik der deutschen Sprache, S. 86-87, 91 (wie Anm. 2).

18 Mit IDS-Grammatik, wobei IDS für Institut für Deutsche Sprache - Mannheim steht, ist die Grammatik von Zifonun et al. (wie Anm. 2) gemeint.

19 Das Vorhandensein eines Subjekts wird nicht gefordert. Für das Deutsche ist damit empirisch zu begründen, dass auch Sätze ohne Subjekt möglich sind, wie etwa mich friert, mir schwindelt, usw. 
fungieren“20. Engels Definition nähert sich dem strukturalistischen Begreifen der Sprache, denn seiner Vorstellung nach wird der Satz aus unterschiedlichen Bestandteilen gebildet, die zusammen eine Struktur bilden. Daraus lässt sich schlussfolgern, dass der Satzcharakter allen Sätzen, die kein finites Verb aufweisen, abgestritten wird. Neben diesem auf den Regeln der Syntax gestütztes Konzept wird der Satz bei Engel weiterhin auch in seinem funktionalen Aspekt beschrieben und ihm somit eine kommunikative Funktion ${ }^{21}$ zugeschrieben: „Der Satz als sprachliches Konstrukt eignet sich in besonderer Weise zur Vereindeutigung von Sprechakten“22.

Abschließend steht die Analyse der Satzdefinitionen aus, die der DudenGrammatik zugrunde liegen. Gleich im ersten Abschnitt des Kapitels Was ist ein Satz? wird vorweggenommen, dass hinter der Bezeichnung Satz „nicht ein einzelner Begriff, sondern eine ganze Familie sich überschneidender Begriffe

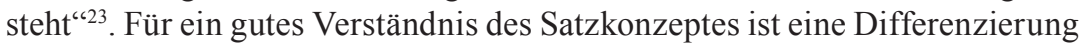
also entscheidend.

Der erste Definitionsansatz orientiert sich an der syntaktischen Struktur des Satzes: „Sätze sind weitgehend vom finiten Verb bestimmt bzw. von dem damit gebildeten Prädikat.“" ${ }^{24}$ Die gleiche Grammatik ergänzt die oben genannte Satzdefinition durch die folgende: „Ein Satz ist eine Einheit, die aus einem Prädikat mit finitem Verb und allen zugehörigen Satzgliedern besteht. “25 Diese Bestimmung gilt für den prototypischen Satz. Die neunte Auflage der Dudengrammatik aus dem Jahr 2016 bietet ferner folgende Definition des Satzes an, die als rein syntaktisch bezeichnet werden kann: „Ein Satz ist die größte Einheit, die man mit den Regeln der Syntax erzeugen kann. ${ }^{\text {"26 }}$ Diese Definition lehnt sich insbesondere an die Satzdefinition von Bloomfield an, der den Satz nach Prinzipien des amerikanischen Strukturalismus als größte selbstständige syntaktische Form, die durch keinerlei grammatische Konstruktionen ihrerseits in eine größere syntaktische Form eingebettet ist, definierte ${ }^{27}$.

20 Engel: Deutsche Grammatik, S. 180 (wie Anm. 9).

21 Der kommunikative Charakter des Satzes wird in der Satzdefinition von Hermann Paul zum ersten Mal in der Geschichte der Syntaxforschung hervorgehoben (vgl. Hermann Paul: Prinzipien der Sprachgeschichte. Tübingen 1970, S. 121).

22 Engel: Deutsche Grammatik, S. 180-183 (wie Anm. 9).

23 Duden: Die Grammatik: unentbehrlich für richtiges Deutsch, S. 775 (wie Amn. 10).

24 Ebd.

25 Ebd.

26 Ebd., 776

27 Vgl. Hadumod Bußmann (Hg.): Lexikon der Sprachwissenschaft. Stuttgart 2008. 
Wie es bei den in Abschnitt 2.1 herangezogenen Grammatiken des Gegenwartsdeutschen der Fall ist, wird die Definition des Satzes in der neueren Grammatikographie des Italienischen seit den 90er Jahren des 20. Jahrhunderts untersucht, damit sich der kontrastive Vergleich auf zeitgenössische grammatische Quellen stützen kann.

Schwarze beschreibt den Satz aus formaler sowie funktionaler Perspektive. Die Form und die Funktionalität sind die zwei Prinzipien, auf denen seine Grammatik der italienischen Sprache beruht. Aus den beiden Prinzipien folgt, dass jede Sprache eine typologisch geprägte Struktur hat und Ausdrucksmittel für die Erfüllung von Bedürfnissen menschlicher Kommunikation und Kognition bereitstellt ${ }^{28}$. Der Satz ist nach Schwarze ,ein Ausdruck, der ein finites Verb enthält und der in seiner formalen und semantischen Struktur um dieses finite Verb herum organisiert ist.“" ${ }^{29}$ Die Elemente, die der (einfache) Satz außer dem Verb enthalten kann, sind nach ihrer inneren Struktur als Konstituenten analysierbar. Bei dieser Definition fällt die wichtige Rolle des finiten Verbs beim Aufbau und bei der Bedeutungsstruktur des ganzen Satzes ins Auge. Das finite Verb konstituiert die Satzstruktur durch seine Argumente, die ihrerseits die Bedeutungsstruktur des Satzes als Verhältnis zur Wirklichkeit zum Ausdruck bringen.

Die formale Bestimmung spielt in der Diskussion des Satzbegriffs auch in der Grande Grammatica Italiana di Consultazione von Renzi et al. weiterhin eine zentrale Rolle ${ }^{30}$. Renzi et al. stellen keine konkrete Definition des Satzes auf; sie betrachten ihn nämlich als ,l'unità massima in cui vigono delle relazioni di costruzione“" (,die größte Einheit, in der Aufbaurelationen gelten“) ${ }^{31}$. Die Autoren orientieren sich grundsätzlich an der internen syntaktischen Struktur des Satzes, bei der das finite Verb durch seine Valenz eine grundlegende Rolle spielt $^{32}$. Daraus lässt sich schlussfolgern, dass diese auf die interne Syntax ausgerichtete Bestimmung den Satz als syntaktische Kombination von Prädikat und Argument(en) zusammenfasst.

Die Position, den Satz als Ergebnis syntaktischer Relationen zu konzipieren, scheint tatsächlich in der Grammatikschreibung des Gegenwartsitalienischen weit verbreitet zu sein. Salvi/Vanelli teilen dasselbe Satzkonzept mit Renzi et al., das als stark formal ausgeprägt bezeichnet werden kann. Sie betrachten den Satz weiterhin als ,la sequenza massima in cui vigono delle

29 Ebd., S. 5.

30 Renzi et al.: Grande grammatica italiana di consultazione (wie Anm. 11).

31 Ebd., S. 29, Übersetzung des Autors.

32 Vgl. ebd., S. 30-31. 
relazioni di costruzione“ (,,die größte Folge, in der Aufbaurelationen gelten“) ${ }^{33}$. Der einzige Unterschied mit der Definition des Satzes von Renzi et al. liegt darin, dass Salvi/Vanelli den Satz nicht als ein Ganzes, sondern als eine Folge (sprachlicher Zeichen) konzipieren. Wie es auch bei Renzi et al. der Fall ist, wird dieser erste Definitionsansatz dadurch ergänzt, dass der (einfache) Satz aus dem finiten Verb und seinen Argumenten besteht ${ }^{34}$. Der Satz ist demzufolge durch eine innere syntaktische Struktur gekennzeichnet, die vom finiten Verb bestimmt wird: Das finite Verb eröffnet um sich herum Stellen für weitere Bestandteile des Satzes, d.h. für seine Argumente, die zusammen zum Ausdruck eines bestimmten Sachverhalts dienen. Salvi/Vanelli (2004) orientieren sich bei der Satzdefinition daher an der syntaktisch-semantischen Bestimmung.

Serianni betrachtet den Satz aus formal-syntaktischer und funktionaler Perspektive. Der Satz wird bei ihm folgendermaßen definiert: „l'unità minima di comunicazione dotata di senso compiuto, che comprende almeno un predicato nella forma di un verbo di modo finito e da un soggetto“ („,die minimale Kommunikationseinheit mit vollständigem Sinn, die mindestens ein Prädikat in der Form eines finiten Verbs und ein Subjekt enthält" $)^{35}$. Der formal-syntaktische Standpunkt geht in der Satzdefinition von Serianni insbesondere von der Forderung nach einer finiten Verbform und einem Subjekt aus. Der funktionale Standpunkt entsteht aus der Erkenntnis, dass der Satz eine bestimmte kommunikative Funktion aufweist; Sätze werden geschrieben/geäußert, weil mit ihrer Hilfe (kommunikative) Ziele beim Leser/Gesprächspartner erreicht werden sollen.

Aus den gesammelten Satzdefinitionen geht deutlich hervor, dass der Satz in der neueren Grammatikographie beider Vergleichssprachen aus unterschiedlichen Perspektiven betrachtet bzw. beschrieben wird. Dies hat zur Folge, dass sich der Satz als eine komplexe sprachliche Erscheinung erweist, die eine begriffliche Systematisierung braucht. Der Vorschlag zu einer allgemein gültigen sprachübergreifenden Definition des Satzes wird im nächsten abschließenden Abschnitt thematisiert.

\section{Abschließender kontrastiver Vergleich und Fazit}

Wie bereits erläutert, wird der Satz in den neueren Grammatiken beider Vergleichssprachen aus unterschiedlichen Perspektiven definiert: aus formal-syntaktischer, semantisch-syntaktischer und funktionaler Perspektive.

33 Salvi/Vanelli: Nuova grammatica italiana, S. 17 (wie Anm. 12), Übersetzung des Autors.

34 Vgl. ebd., S. 27.

35 Serianni: Grammatica Italiana, S. 85 (wie Anm. 14), Übersetzung des Autors. 
Die meisten Grammatiken, die für die Zielsetzung des vorliegenden Artikels ausgewählt wurden, definieren den Satz aus einem formal-syntaktischen Standpunkt heraus; der Satz wird meistens als sprachliche Einheit definiert, die aus Subjekt und Prädikat besteht ${ }^{36}$. Eine allgemein akzeptierte vollständige Satzdefinition, die von den drei oben erwähnten Perspektiven ausgeht, ist in der Grammatikographie beider Vergleichssprachen nicht zu finden. Daraus entsteht der Vorschlag dieses Artikels zu einer wissenschaftlich allgemein akzeptierten Satzdefinition; um dieses Ziel zu erreichen, soll der Satz aber als (grammatische) Kategorie ${ }^{37}$ angesehen werden, die formal-syntaktische, semantische und funktionale Kriterien ausmachen, wie die folgende Abbildung überblickshalber zusammenfasst:

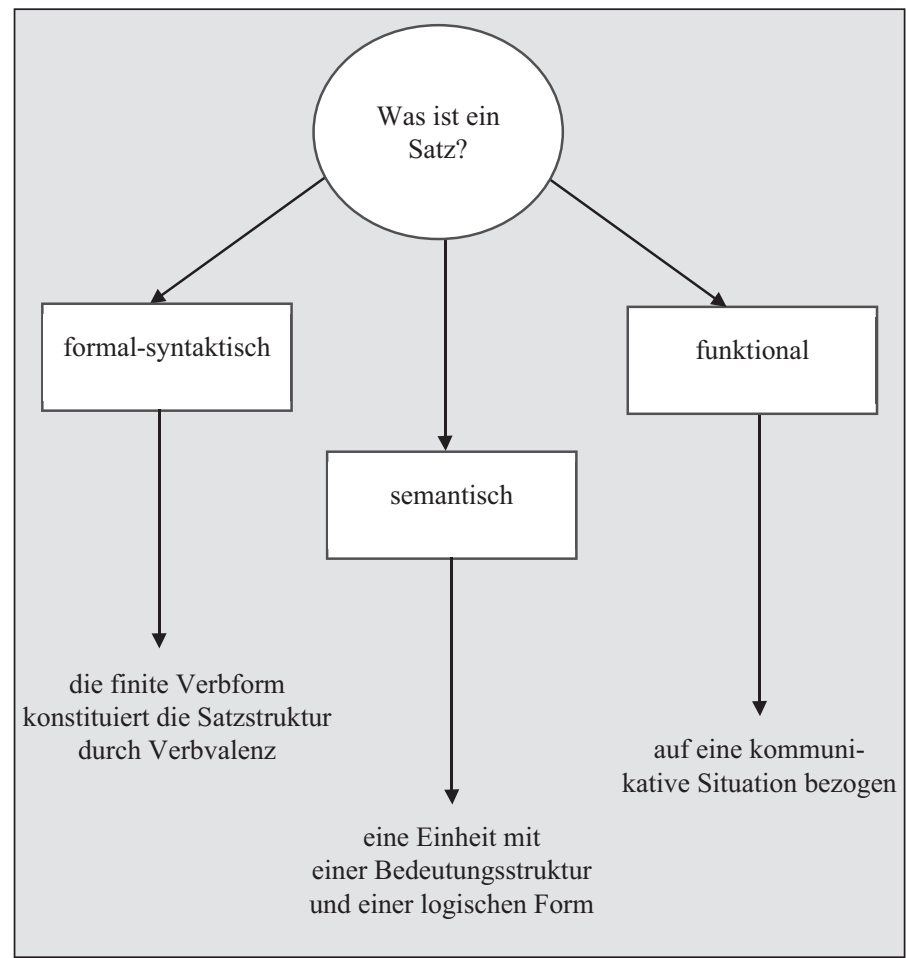

Abbildung 1: Merkmale des Satzes als Kategorie

36 Nach Christa Dürscheid geht diese Satzdefinition auf die aristotelische Logik zurück (Christa Dürscheid: Syntax. Grundlagen und Theorien. Göttingen ${ }^{3} 2005=$ Studienbücher zur Linguistik. Hrsg. von Peter Schlobinski. Bd. 3).

37 Der Satz wird als Kategorie genauso wie die Wortarten traditioneller Grammatiken wie Verb, Substantiv, Adjektiv, Adverb, Präposition, Partikel, Konjunktion, Partikel und 
Die in der Abbildung 1 enthaltenen Kriterien, auf deren Grundlage der Satz als Kategorie definiert werden kann, gelten für beide Vergleichssprachen (Deutsch-Italienisch); aus der Feststellung, dass das Konzept Satz übereinzelsprachliche Geltung hat, wird im Folgenden der Vorschlag gemacht, ein für die beiden Zielsprachen allgemein gültiges Profil für die Satzdefinition abzubilden, wofür formal-syntaktische, semantische und funktionale Kriterien die Grundlage bilden:

Ein (typischer) Satz enthält eine finite Verbform und die von ihr geforderten Komplementen (Syntax). Im Zusammenspiel von Verb und den von ihm verlangten Komplementen wird ein bestimmter Sachverhalt zum Ausdruck gebracht (Semantik). Jeder Satz ist auf eine konkrete kommunikative Funktion bezogen (Funktionalität/Pragmatik).

Abschließend soll unterstrichen werden, dass dieselbe sprachübergreifende grammatische Satzdefinition dazu dienen soll, den Sprachvergleich (DeutschItalienisch) in der DaF-Didaktik zu fördern. So kann sie beispielsweise als Ausgangspunkt für kontrastiv ausgerichtete syntaktische Untersuchungen, mit besonderer Rücksicht auf den Satzbau und daher auf die Wort- bzw. Verbstellung im Satz dienen.

\section{Literaturverzeichnis}

Bußmann, Hadumod (Hg.): Lexikon der Sprachwissenschaft. Stuttgart 2008.

Duden: Die Grammatik: unentbehrlich für richtiges Deutsch. Band 4. Berlin ${ }^{9} 2016$.

Dürscheid, Christa: Syntax. Grundlagen und Theorien. Göttingen ${ }^{3} 2005$. (=Studienbücher zur Linguistik. Hrsg. von Peter Schlobinski. Bd. 3)

Eisenberg, Peter: Grundriss der deutschen Grammatik. Stuttgart 1989.

Engel, Ulrich: Deutsche Grammatik. München 2009.

Heringer, Hans Jürgen: Lesen, lehren, lernen: Eine rezeptive Grammatik des Deutschen. Tübingen 1989.

Heringer, Hans Jürgen: Theorie der deutschen Syntax. München 1970. (=Linguistische Reihe 1)

Müller, Beat Louis: Der Satz. Definition und sprachtheoretischer Status. Tübingen 1985. (=Reihe Germanistische Linguistik 57)

Paul, Hermann: Prinzipien der Sprachgeschichte. Tübingen 1970.

Renzi, Lorenzo/Salvi Giampaolo/Cardinaletti Anna (a cura di): Grande grammatica italiana di consultazione. Volume I. La frase. I sintagmi nominale e preposizionale. Bologna 2001.

Pronomen betrachtet. Das System der grammatischen Kategorien dient der Klassifikation des Wortbestands einer Sprache in wenige Wortklassen, die bestimmte Eigenschaften gemeinsam haben (Peter Eisenberg: Grundriss der deutschen Grammatik. Stuttgart 1989, S. 34). 
Ries, John: Was ist ein Satz? Prag 1931.

Salvi, Giampaolo/Vanelli Laura: Nuova grammatica italiana. Bologna 2004.

Schlobinski, Peter: Funktionale Grammatik und Sprachbeschreibung. Eine Untersuchung zum gesprochenen Deutsch sowie zum Chinesischen. Wiesbaden 1992.

Schröder, Thomas: Die Handlungsstruktur von Texten. Ein integrativer Beitrag zur Texttheorie. Tübingen 2003.

Schwarze, Christoph: Grammatik der italienischen Sprache. Tübingen 1995.

Seidel, Eugen: Geschichte und Kritik der wichtigsten Satzdefinitionen. Jena 1935.

Serianni, Luca: Grammatica Italiana. Italiano comune e lingua letteraria. Novara 2006.

Zifonun, Gisela/Hoffmann Ludger/Strecker Bruno et al.: Grammatik der deutschen Sprache. Berlin 1997. 